\title{
Study on Application of IOT in the Cotton Warehousing Environment
}

\author{
Jia Jiang, Donghai Yang and Zhe Gao \\ School of Economics and Management, Hebei University of Science and \\ Technology, Shijiazhuang 050018, China \\ jiang_jia67@163.com
}

\begin{abstract}
Cotton is an important strategic material for national well-being and the people's livelihood. The warehousing environmental conditions will directly affect the storage quality of cotton or economic value, and even have some influence on the whole national economy. In order to improve the relatively out-dated management of Chinese cotton warehousing industry, this paper firstly analyzed the existing problems and the needs of future development in the management of China cotton warehousing industry, and then constructed the IOT architecture suitable for cotton storage based on the advantage of IOT technology, and finally designed detailed IOT application solution of cotton warehousing with specific examples from such technology application layers as sensing layer, network layer, and application layer, which aims to make an useful attempt to enhance the management level of China cotton warehousing.
\end{abstract}

Keywords: IOT technology, cotton, warehousing environment, application

\section{Introduction}

\subsection{The Current Situation of Warehouse in China's Cotton Industry}

China is an important cotton production country in the world, and its cotton production, consumption, imports and exports of cotton textiles rank first in the world. Cotton industry has played an important role in the development of our national economy. At present, China cotton planting area reaches 70 million mu with an annual output of about 7 million tons of lint. The main cotton producing area locates in the region of Xinjiang, the Yellow River and the Yangtze River basin. The import of cotton mainly concentrated in the Qingdao Port and Shanghai Port, which accounts for about 70\%-80\% of the total national imports. The sales of cotton are mainly distributed in the Yangtze River Delta region, North China region and Hubei and Hunan provinces with the center of Wuhan. This kind of cotton production and sales distribution requires an effective coordination mechanism to ensure that Xinjiang Cotton and imported cotton can be transported to inland areas timely and accurately, which may realize effective displacement of cotton from production regions to processing and consumption areas. Thus the cotton storage becomes the focus of the cotton quality control. Because of late market reform of the cotton management system and other historical reasons, China cotton management has many problems in the warehouse management and the application of information technology on warehousing, which directly affects the economic and social benefit of cotton logistics. The main problems are as follows: the manual code division and household bookkeeping manner in the cotton warehouse management may result in cotton warehousing information omission or rerecording. This may cause incomplete statistics of basic information such as cotton batch, and the sharing of information resources can not be realized accurately; the traditional temperature and humidity meters are still used to test and record cotton temperature and humidity, so the data collected is 
discontinuous and incomplete, the history condition can not be recorded exactly and the historical data can not be analyzed and mined accurately, let alone the intelligent early warning for the quality of cotton storage; the monitoring of cotton safety mainly focus on fire alarming system, which most adopts smoke detecting cable technology for fire sensor network cabling, which has such problems as complicated wiring, poor expansion of communication mode, high rate of the system failure and false alarming, especially the aging of wires may result in electric leakage or even fire; cotton warehouse still adopts manual inspection of cotton storage environment. Although the traditional and outdated video device may be equipped, it has no effective relation to the central control system, therefore cotton storage environmental visualization management cannot be realized and the history of accident cannot be traced back.

\subsection{The Development Trend of Warehouse Management in China's Cotton Industry}

In order to meet the development requirements of the new economic situation, the management level should be improved in the cotton industry in order to play its important role in the national economy system. To achieve this goal, the informationization construction should be strengthened based on the characteristics of the industry. It also should be promoted in the aspects of information source, channel and processing platform with advanced technology. The Internet of things which features the overall perception, reliable transmission and intelligent processing has become the important promoter of cotton industry informatization construction. With the application of the cloud computing, 4G network, mobile Internet, intelligent sensors and other high-tech in the cotton warehousing operations, the cotton IOT architecture will be constructed with economical, practical and efficient features. Therefore, the visualization of the process of cotton logistics management and the intelligent control of quality and safety would be achieved to serve the national economy system efficiently.

\section{Brief Introduction to IOT (The Internet of Things)}

IOT refers to a kind of network for real-time information exchange and communication, in which items will be combined with the Internet by means of various modern information sensing equipments and agreed protocol in order to achieve comprehensive management of intelligent identification, location, tracking, monitoring.

\subsection{Technical Architecture of Internet of Things}

As an important part of a new generation of information technology, the Internet of things is a new system for real-time interaction between virtual network and the real world. It is extended on the basis of the Internet, and its key function is to achieve comprehensive perception and control for things. Internationally recognized IOT structure contains three levels: sensing layer, network layer and application layer. As shown in figure 1 .

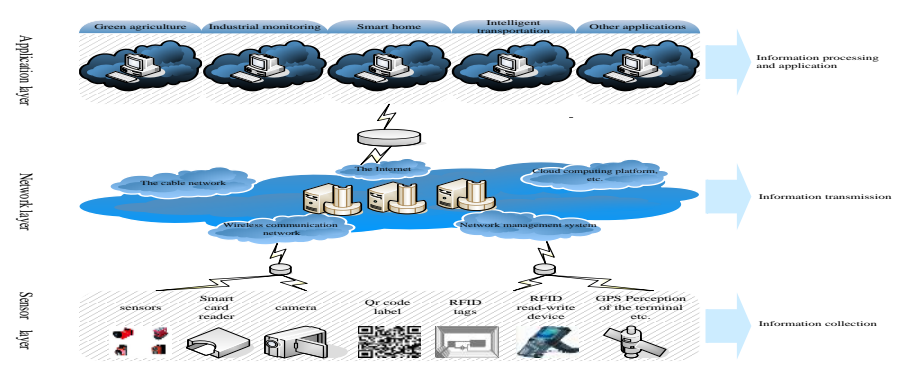

Figure 1. IOT Technology Architecture 
The function of sensing layer is to achieve a comprehensive, intelligent perception by obtaining the data of the physical world. It includes such sensing terminals as a twodimension code tag, RFID tag, reader, camera, GPS and other various sensors; the network layer connects the sensing layer and the application layer. It usually consists of wired communication network, wireless communication network, the Internet, network management system and the cloud computing platform; as the deep integration product of the information technology and industry environment, application layer mainly refers to the deep mining the related data in order to support scientific decision of the system. It has been widely used in the smart home, telemedicine, airport intrusion detection systems, intelligent transportation, and environmental monitoring. It is the interface between the Internet of things and the user.

\subsection{The Key Technology of the Internet of Things}

The key technology of IOT involves mainly in the field of sensing technology, network communication technology and data processing technology.

(1) Sensing technology

Sensing technology, i.e. information acquisition technology, mainly refers to basic information collection and storage in the physical world. At present the mainstream ways of collecting information include electronic label and sensor acquisition. Electronic label is used for standardized identification of sensing information, and meanwhile it can read and identify information by the reading and writing device of RFID and bar code. The sensor is used for sensing measurements, such as temperature, humidity region, light and other environmental parameters, and providing the original basic information for later intelligent decision of IOT application layer.

(2) Network communication technology

IOT communication technology refers to the communication between people and objects, objects and object, the physical world and the real world. The transmission of sensing information can be constructed with wired or wireless technology according to the actual situation. The wireless communication technology represented by the ZigBee technology was mostly used in the IOT technology application. ZigBee technology [1] is based on the IEEE802.15.4 standard, used for two-way wireless communication technology for the data transmission among various electronic equipments with short distance, low data rate, low cost of transmission and for periodic, intermittent, low response time data transmission.

(3) Data processing technology

The data processing technology usually indicates that data is collected by computer and then processed to generate new forms of information. Data processing is divided into the steps of data acquisition, data conversion, data packet, data organization, data calculation, wherein the data calculation is especially important. And currently the data calculation is mainly based on the cloud computing technology. The advantage of cloud computing lies in the capability to split the huge computer processing program automatically over the network into numerous smaller units of the subroutine, and then process the subprogram in the huge system composed of multiple servers [2]. Relying on the cloud computing technology, network service providers can process tens of millions of information in short time and even in a few seconds, just as powerful as supercomputer with the service of network operation analysis [3-4]. Cloud computing, with its excellent data processing, storage, analysis ability, will become the solid foundation for the future development of the Internet of things.

Therefore, the Internet of things has been used more and more widely in many related fields with the advantages of extensive data sensing, safe and reliable information transmission and intelligent information processing. In the application process the IOT 
architecture should be built by the appropriate technical means based on the characteristics of the environment. Meanwhile, the economical and proper equipment, apparatus and execution system should be developed to ensure the safe and efficient operation of the entire Internet of things [5-8].

\section{Design for the Application of IOT in the Cotton Storage}

Study on Application of IOT in cotton warehousing mainly focuses on cotton static long storage, flammable feature and quality degradation easily affected by the environment.

\subsection{IOT Architecture for Cotton Storage}

The IOT architecture design of cotton storage is based on business process analysis of cotton storage and library management with the introduction of the general networking model into cotton storage.

(1) Business process of Cotton storage

Business process of cotton storage consists of three stages of check-in, warehousing and check-out. The specific business activity and information flow are shown in figure 2 .

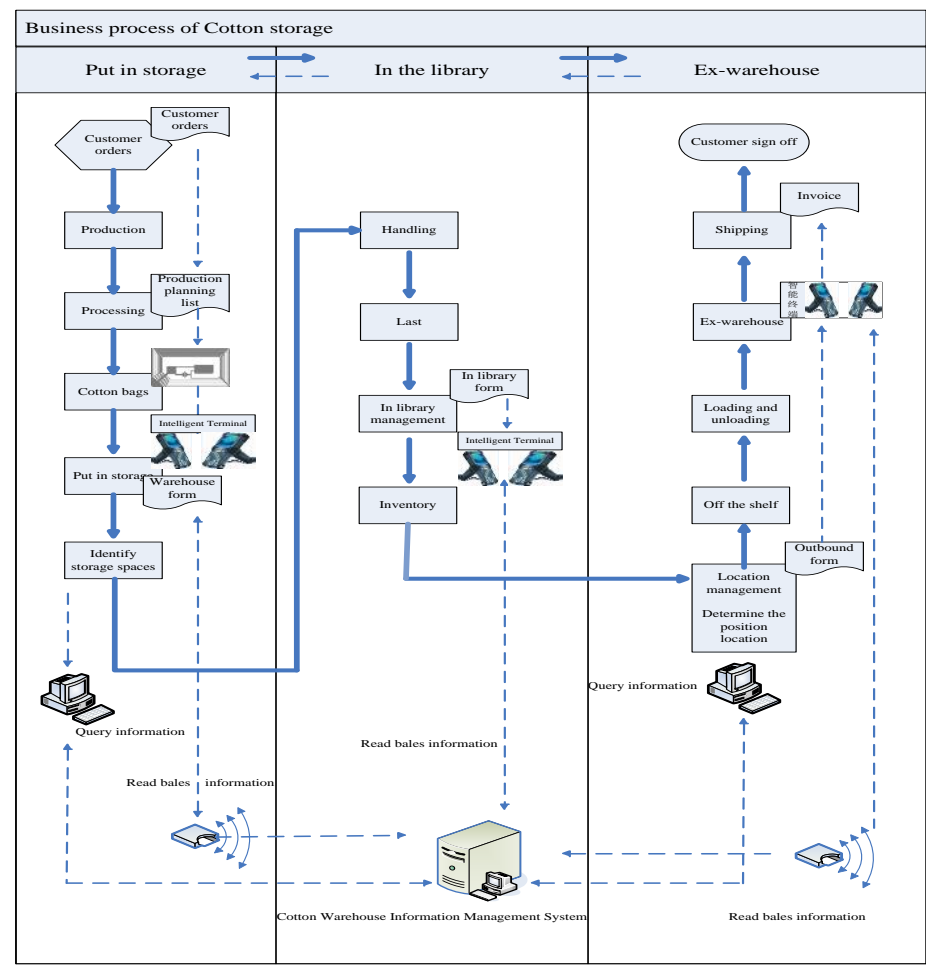

Figure 2. Business Process of Cotton Storage

With the successful application of information technology in the cotton storage business, how to build IOT network with "comprehensive sensing, interconnection" for cotton storage has become the consensus of experts and scholars in the cotton fields of, and relative research on key technology has been conducted.

(2) IOT architecture of cotton storage based on business process

Cotton storage business process based on the cotton warehouse, combined with the current actual situation, in-depth analysis of modern logistics information technology will be used in each link, in order to improve cotton warehousing information management level, realize real-time monitoring and control decision-making capacity storage 
environment as the goal, we design a IOT of basic framework of cotton [9]. Cotton IOT mainly includes three main parts: sensing information collection and storage of temperature and humidity in the front end, safe transmission of sensing and monitoring information in the middle and application demonstration system for monitoring cotton storage characteristics in the end. The overall architecture of cotton IOT is shown in Figure 3.

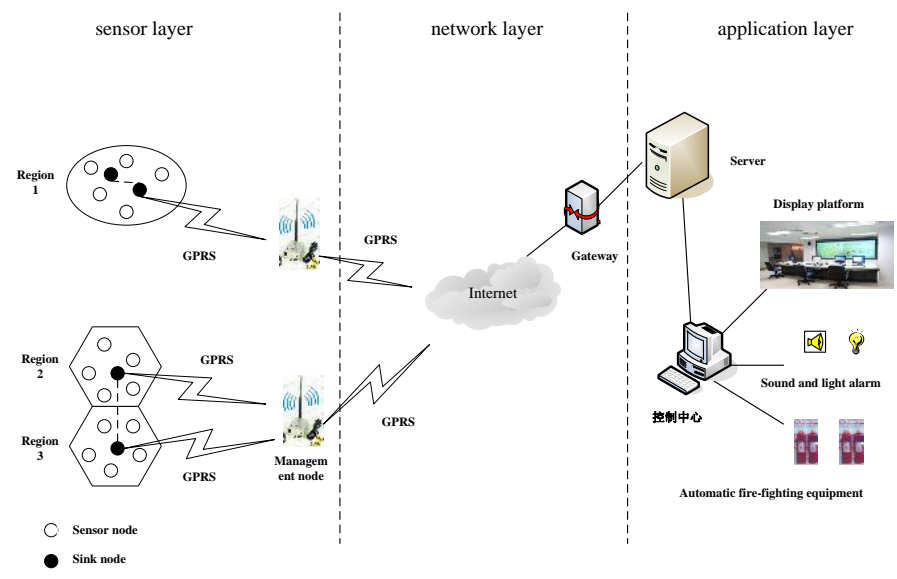

Figure 3. Cotton IOT Architecture

The front-end data acquisition network adopts ZigBee tree network, composed of sensor nodes, sink nodes and management nodes. In the deployment of the system, some sink nodes, are firstly fixed in the monitoring area. The sink node is the router in the unit area, gathering network information. Sensor nodes can be random arranged based on actual needs. Therefore, the sensor nodes will keep transmitting the sensing data of temperature and humidity to the sink node, and then the sink node transmits to the management node. Middle management node will transmit the data information of temperature and humidity to the control center through GPRS packet switching technology and the Internet. The terminal control center has access to the data on the network server, real-time monitoring the basic information of temperature and humidity collected by different sensors in different regions and conducting systematic analysis of historical data. It aims to analyze and judge the number, abnormal temperature and humidity, smoldering and other issues in the cotton storage so as to form the basis for decision making.

\subsection{IOT Technology of Cotton Storage}

According to the basic IOT structure of the cotton storage, we would put forward the specific technical scheme respectively for the perception layer, network layer and application layer.

\subsubsection{The Sensing Layer}

Warehousing environmental conditions may directly affect the quality of cotton storage, or the economic value of stored cotton, and cotton storage security. Therefore, the primary task of controlling cotton storage quality is to develop the front-end monitoring system obtaining the real-time environmental changes of cotton storage. The front-end monitoring system for cotton warehousing environment mainly includes three parts of monitoring equipment, the front-end gateway and networking protocol.

(1) Selection and development of monitoring equipment. 
The physical characteristics of storage environment, especially temperature, humidity, have great effects on cotton quality and security. Therefore, we need to develop monitoring equipment for sensing and acquiring the physical environment information of cotton warehouse, including temperature sensor, humidity sensor etc. At the same time, in order to facilitate the staff to monitor remotely and discover in time the abnormal conditions of the cotton warehouse environment, such as fire aura of cotton, we also need to develop a video sensor with interaction control, conducting real-time, continuous monitoring of the cotton warehouse environment.

(2) The front-end gateway development.

The monitoring data of various sensor equipment monitoring cotton warehousing environmental should be converged and transmitted to the background display system. Therefore we need to develop front-end gateway for collecting and transmitting monitoring data of sensors. The gateway with some communication interfaces, connected with various monitoring equipment, can collect and converge monitoring information from the monitoring equipment, and transmit timely to the background display system. At the same time, gateway should support the implementation of pretreatment operation for some monitoring data, such as abnormal data filtering and video compression coding in order to reduce the data redundancy and improve transmission efficiency.

(3) Development of network protocol.

Because of huge monitoring area of the cotton warehousing environment and limited communication distance of the monitoring equipment, usually with no direct communication with the front-end gateway, it is necessary to establish the front sensing network with self-organizing and multi hop among various monitoring equipment in order to form a complete front-end monitor system between monitoring equipment and gateway [10].The network protocol should be developed in the establishment of a front-end sensor network. Network protocol will be addressing, addressing the sensors and gateways, independent networking and routing, adapt to the dynamic change of the network topology so that it has the capacity to find new monitoring equipment and tolerate the failure of monitoring equipment, and also support the reliable and real-time transmission of monitoring data.

\subsubsection{The Network Layer}

The network layer is mainly related to the remote acquisition and storage technology for the environmental monitoring information of cotton warehousing and secure transmission technology for the monitoring information of cotton warehouse.

(1) Remote acquisition and storage of monitoring information for cotton warehousing environment

Technical research on remote acquisition and storage of monitoring information for cotton warehouse.The monitoring data distributed in different cotton warehouse should be converged to the background display system through remote transmission. Because of the multi-category of the monitoring information for cotton warehouse, the research on acquisition and storage technology for monitoring information of cotton warehousing environment should be conducted: in order to reduce the data redundancy and bandwidth, and ensure real-time and priority transition for important monitoring data, data filtering and different types of information fusion should be developed based on the importance of data feature collected by monitoring equipment; because of the different types of monitoring information for cotton warehouse, such as temperature, humidity, video, etc. there are significant differences in data formats, numerical range and semantic aspects for different types of monitoring information. Therefore, the storage of monitoring information for cotton warehouse needs to take into account the characteristics of sensory data of different types of monitoring equipment, and data 
standard and metadata standards should be established in order to store and index the monitoring information.

(2) Safe transmission of monitoring information for cotton warehouse

Technical research on safe transmission of monitoring information for cotton warehouse [11-15]. Cotton reserve information relates to national strategic security, so any form of information disclosure are very likely to cause irreparable loss to national cotton strategic reserve. Therefore, we must guarantee the transmission security of monitoring information acquired by the front-end sensing equipment. Specifically, encrypted monitoring information of the front end transmits to the control center, which can effectively prevent the monitoring information leaks in the transmission process.

\subsubsection{Application Layer}

The research in the application layer includes the development of information demonstration system for cotton warehousing environmental monitoring, the control technology of limited user access for cotton warehouse environmental monitoring information and intelligent early warning for the quality and safety of the cotton warehouse.

(1) Information display system of cotton warehousing environmental monitoring. The background demonstration system development of cotton warehouse monitoring information mainly realize the real-time display of monitoring information and tracing analysis of historical data. Real time display is to realize visualization of the cotton warehouse environmental monitoring information, displaying all types of real-time monitoring information of cotton warehouse environment. Specifically, evaluating the importance of monitoring information from different sensing sources; marking automatically the priority of monitoring information of sensing sources; and displaying unexpected abnormal data and information for the user, at the same time, for the switching of sensing sources, demonstration system can conduct continuous, stable transmission and real-time display of the monitoring information with no influence of the warehouse location, transmission distance and other factors, achieving smooth switching for multi sensing sources. The historical data analysis is to index the cotton warehouse environment monitoring history information, obtaining the changing laws of cotton storage environment for the corresponding regions to support the government to make scientific decision.

(2) Limited user access control technology for cotton warehouse environmental monitoring information [16-17]. Specifically, the background display system implements the classification access to monitoring information. All users need to pass strict verification of user authentication to have access to monitoring information, and only can the authorized user have access to the monitoring information with different authority.

(3) Intelligent early warning for the quality and safety of cotton storage. According to the changing laws of fire, quality (such as cotton fiber length), we establish the corresponding data analysis model, develop early warning software and implant into the display system. Through the front-end monitoring equipment acquisition and safe transmission, the central database can make systematic analysis of basic information, such as cotton temperature and humidity, to realize the intelligent early warning on the quality and security of cotton storage.

\section{Case of Application}

In order to validate the feasibility of the project, we demonstrate our scheme in the standardized cotton warehouse respectively in CNCGC and the local Cotton Corporation under CNCGC. The demonstration mainly focuses on the library management module in the cotton warehousing process, mainly testing the environment safety and quality management system of cotton warehousing. The display system of environmental safety 
monitoring and quality management in the cotton warehousing is divided into central and local levels. The central control system is arranged in CNCGC, collecting occurred or predicted data in every local cotton warehouse and giving orders in order to real-time monitoring information of local warehouse environment. Local control system is arranged in a representative standardized cotton warehouse of local Cotton Corporation under CNCGC, the front-end monitoring equipment and back-end systems of cotton warehouse are arranged to monitor the cotton warehouse characteristics including temperature, humidity and other indicators, which provides real-time acquisition and monitoring of warehouse environment information in the local warehouse.

\subsection{Deployment and Application of Front-End Monitoring Equipment of Cotton Warehouse}

In the selected standard cotton warehouse, 2 smoke sensors are fixed in the top ends of the warehouse, collecting information with fire characteristics; 2 high precision tracking cameras are fixed at both ends of the cotton cross channel with a distance of 1-1.5 meters away from the roof, collecting video data; 2 integrated temperature and humidity sensors (independent development) are fixed respectively in the typical positions of top, middle and lower levels of the warehouse, collecting the temperature and humidity data. The sensors used should strictly meet the criteria of the national security standard of cotton warehousing industry, and they are powered by weak power supply or a self-contained power supply in order to avoid cable wiring which may have a fire crisis. The sensor network layout of sensing layer adopted the current mainstream ZigBee tree network, divided into four modules: smoke sensor, temperature and humidity sensor, data acquisition and ZigBee wireless. For the ZigBee tree network structure, each main node not only can be connected with the sensor for data acquisition and monitoring, but also can automatically transmit the data from other network nodes. In addition, each main node also has wireless connection within the scope of coverage with multiple child nodes which don't undertake the task of transferring the network information. The above characteristics of ZigBee tree network can effectively ensure the real-time collection and transmission of the cotton warehouse environmental monitoring data, providing strong technical support for emergency disposal for the daily warehouse management.

\subsection{Design and Application of Backend Systems of Cotton Warehouse}

The backend system was arranged in the corresponding control room of cotton warehouse, responsible for analyzing and predicting the acquisition data, based on data mining technology, from different monitoring equipment, including text, sound, image, video, etc. and for making decision and control for the cotton warehouse events. The central control system was deployed in CNCGC, aiming to realize real-time acquisition, storage, display of the cotton warehouse storage environment information throughout the country, and then made timely processing of emergency in the corresponding area.

\subsection{Encryption Transmission of Sensing Information of Cotton Warehousing Environment}

The specific process of sensing information encryption of cotton warehousing environment is as follows: firstly the cotton sensing information is saved to local database of the cotton warehouse, and then synchronize the local database of cotton warehouse and the central database by using self-developed database synchronization program, thus the central collected database of all the cotton warehouse, so as to achieve the effective query to the information of local cotton warehouse. Database synchronization program consists of server and client of cotton warehouse, and it has been successfully tested through simulation, as shown in figure 4: 

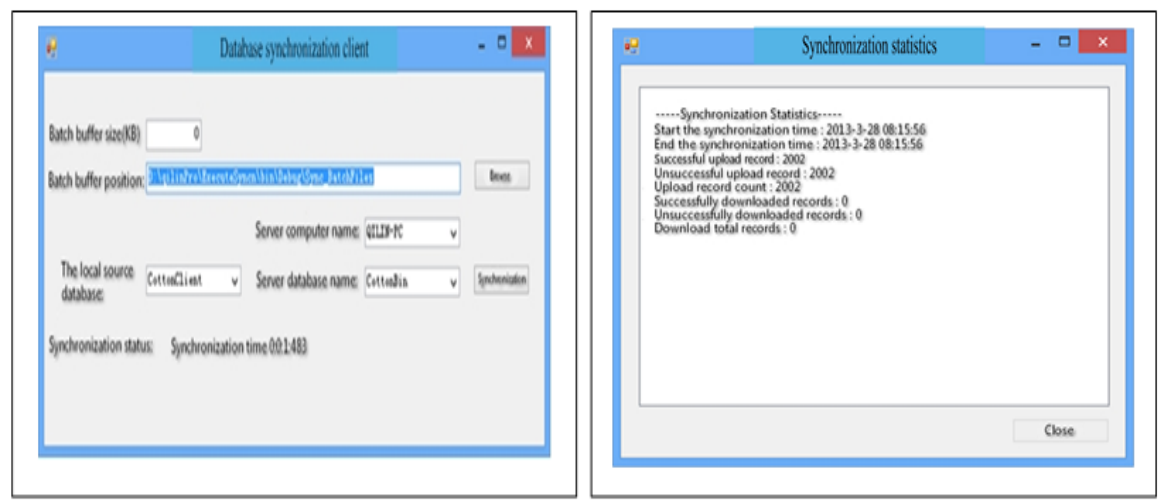

\section{Figure 4. Synchronization Interface of Database Synchronization Client}

\subsection{Encryption storage of sensing information of cotton warehousing environment}

Data stored in the database is not safe. The famous user password of CSDN website was leaked because the user password was saved to the database in plaintext form. The use of storage encryption can effectively prevent stealing or tampering the database file content by OS tools. Even if the hacker steals the key data, it is still difficult to get the required information, because all data are encrypted. In addition, unauthorized system administrators can not see the contents of the data under encryption, greatly improving the safety of key data. Storage encryption was conducted by Microsoft function of encryption, decryption, which would not use the certificate stored in the database and the emergency data of encryption key, it can directly encrypt and decrypt data based on the password supplied by user. The pass phrase (key) is not stored in the database, which means that the system will not be "cracked" by the stored data. Due to the huge amount of sensing information of the cotton warehouse, relevant content of data storage encryption was currently only used in the user password and access control, and it may cover basic information of cotton warehouse, cotton bin, and equipment according to needs in the future.

\subsection{Display System of Environmental Monitoring and Quality Management of Cotton Warehousing}

The display system of environmental monitoring and quality management of cotton warehousing was designed on the basis of $\mathrm{B} / \mathrm{S}$ and $\mathrm{C} / \mathrm{S}$ architecture. The development of front desk adopted B/S mode, while background system C/S mode [18]. Display system is divided into the central monitoring system and local monitoring system. The central monitoring system was for CNCGC, while the local monitoring system mainly distributed in the local cotton corporation in all parts of the country. The functions of central monitoring system include the map display of national cotton warehouses, map display of different regional cotton warehouse, video monitoring of cotton warehouse or bin, the control of temperature and humidity, historical data query to temperature, humidity and video, early warning of quality safety, emergency treatment, etc. The local monitoring system is mainly responsible for video, temperature and humidity monitoring in the local cotton warehouse for timely reporting will-be emergency and the disposal opinions or conclusions. The overall system architecture is shown in figure 5. 


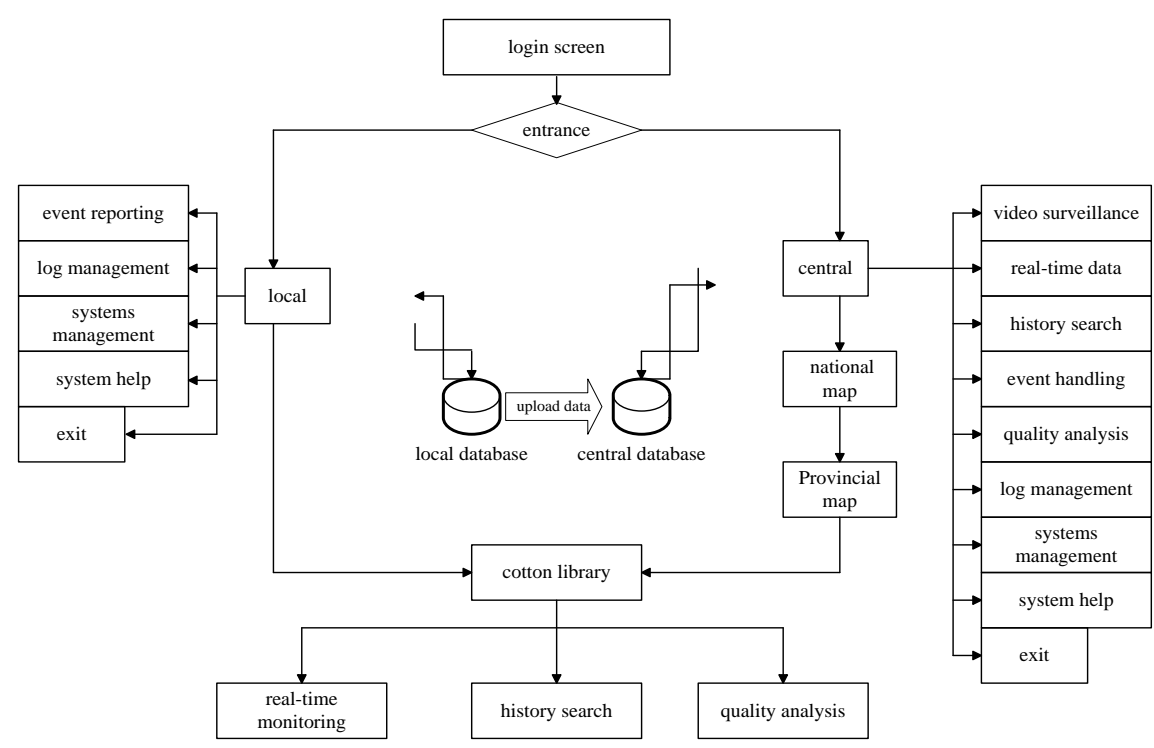

Figure 5. System Architecture of Environmental Monitoring and Quality Management of Cotton Warehousing

\subsubsection{Technology Security Access Control Information}

The C/S mode system is for the central personnel to modify and set the global information, having access and control for the information of all the cotton warehouses, including the generation of character or cotton base administrator, alarm management, equipment management. The local application program is responsible for the local user management, planning of the role, equipment management (device access), and cotton warehouse management. The $\mathrm{B} / \mathrm{S}$ mode system is mainly used for legitimate users to have access to the data. In the $\mathrm{B} / \mathrm{S}$ system, the central personnel have access to information of all the cotton warehouses according to the distribution of authority, while the staff of cotton warehouse only has access to the local display system. Security access control of sensing information mainly covers the two aspects of personnel and equipment.

Personnel access control technology [19]. In the access control technology, B/S and $\mathrm{C} / \mathrm{S}$ adopt different access control mechanism. The B/S mode system employs role based access control (RBAC), which means that the administrator defines a set of characters (roles) and put them to the subject. The system process and ordinary users may have different roles. If an object was set into some type, the subject has the corresponding role to visit it. Thus administrators can avoid the tedious work of defining each user's permissions. C/S system is a kind of access control with multi regions and multi roles. $\mathrm{C} / \mathrm{S}$ is a multi-region and multi-role based access control (RRBAC), which added region based on RBAC. System design includes regional management module, the role management module, subject management module, rights management module and user management module.

Device access control technology. For the device access control technology, all the related devices adopt the access mechanism. Before using the equipment, equipment registration and authorization must be done first, otherwise it cannot be accepted by the system. 


\subsubsection{Display System of Environmental Safety Monitoring Information of Cotton Warehousing (B/S System)}

The main interface of display system for encryption transmission and storage of sensing information and for control mechanism of user security access is shown in figure 6.

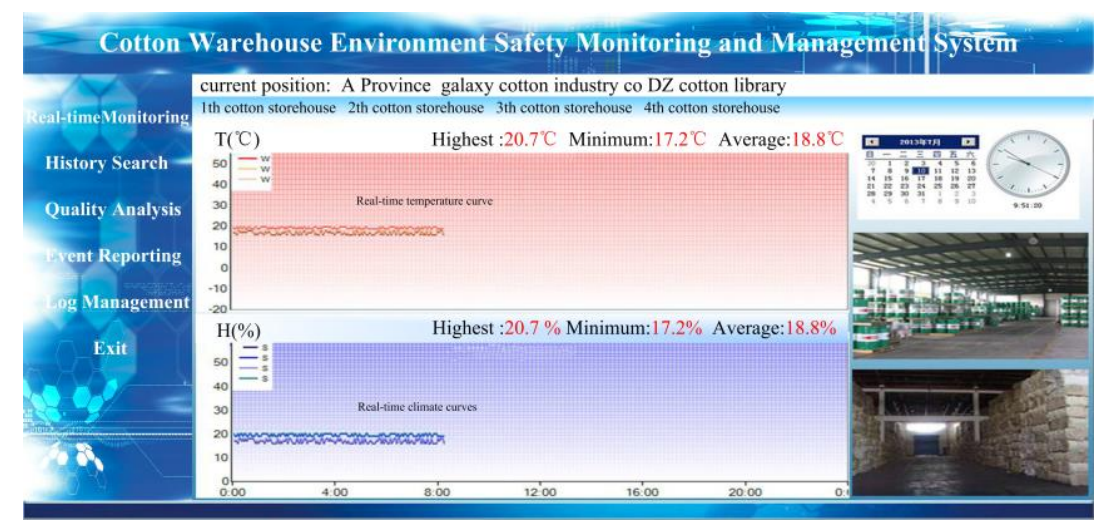

\section{Figure 6. Display System Interface of Environmental Security Monitoring and Quality Management of Cotton Warehousing}

This system is divided into two parts of the central and local. The central monitoring system is for CNCGC; the local monitoring system is for the local cotton corporation limited in various regions of the country. For system management personnel at all levels in accordance with their respective authority and responsibility through interactive control system to can realize scientific control of overall strategy for cotton warehousing industry and effective control of cotton quality and safety of the cotton warehouse field.

\section{Conclusion}

This paper constructed cotton IOT architecture based on the current situation and development trend of the cotton storage and the working principle of IOT, and then put forward the technical scheme according to the environment characteristics of cotton warehousing, which filled the blank field of IOT technology application in cotton storage. Moreover, the field application demonstration of specific scheme was conducted in CNCGC and the cotton warehouse under CNCGC. After a period of the field demonstration, the IOT technology has been effectively applied in cotton warehousing environment, and we have got relatively ideal results, which will provide strong support for the information construction of the follow-up work of the cotton industry.

The successful operation of the front end monitoring system in the field including temperature, humidity, video sensor, front-end gateway and network protocol produced comprehensive, real-time, objective and effective information convergence, which overcome maximum the incomplete and delayed information in the traditional data acquisition mode. It has laid the foundation for the subsequent intelligent analysis and scientific decision, and also may reduce the fire risks caused by traditional manual inspection.

The introduction of data filtering and information fusion mechanism in the local transmission or local to the central transmission for monitoring information of cotton warehousing reduced data redundancy and bandwidth in the data transmission, ensuring the real-time, effective priority data transmission. At the same time, this has effectively avoided leakage problem in the transmission process to ensure information security of the national strategy material. 
The monitoring information display system of cotton warehousing environmental safety has strict storage and access security control technology for perceptual information, realizing the effective control for the permission of management personnel and ensuring storage security of information.

The development and application of monitoring information display system of cotton warehousing environmental safety provided the visualization of all kinds of monitoring information at all levels. With the effective operation of analysis and early warning software for cotton quality and analysis and early warning software for cotton fire embedded in the system, we not only provided early warning system for preventing cotton quality degradation and cotton fire but also provided effective evidence to the design of facilities construction plan for cotton storage to ensure the quality of cotton storage. At the same time, the article also has some shortages: although the sensing information encryption can avoid the leakage of monitoring information effectively, the real-time transmission and smooth switching of video signal have not been implemented fully due to various concurrent events and related limited technology and research conditions. In addition, because there are no mature and authoritative research results for the law of cotton smoldering currently, it is really difficult to achieve accurate and effective early warning for the problem, which should be improved in the future research.

\section{Acknowledgements}

Project Support: Cotton storage environment monitoring information of remote acquisition, storage and display system (National science and technology support plan) (2012BAH20B030-4)

\section{References}

[1] H. Benxiong, L. Yafang, Z. Fan and W. Xin, "Design and analysis of association period of IEEE802.15.4 beacon enabled network", J. Huazhong Univ. of Sci. \& Tech. (Natural Science Edition), vol. 36, (2008), pp. 28-30.

[2] Q. Jin, M. Duoqian and Z. Zehua, "Knowledge Reduction Algorithms in Cloud Computing”, J. Chinese Journal of Computers, vol. 34, (2011), pp. 2332-2343.

[3] K. Chen and W. M. Zheng, "Cloud Computing: System Instances and Current Research", J. Journal of Software, vol. 20, (2009), pp. 1337-1348.

[4] S. Brin and L. Page, "The anatomy of a large-scale hypertextual Web search engine", J. Computer Networks, vol. 30, (1998), pp. 107-117.

[5] L. Atzori, A. Iera and G. Morabito, "The Internet of Things: A survey", J. Computer Networks, vol. 54, (2010), pp. 2787-2805.

[6] R. H. Weber, "Internet of Things-New security and privacy challenges", J. Computer Law \& Security Review, vol. 1, (2010), pp. 23-30.

[7] Y. Jincui and F. Binxing, "Security model and key technologies for the Internet of Things", J. The Journal of China Universities of Posts and Telecommunications, vol. 18, (2011), pp. 109-112.

[8] R. H. Weber, "Internet of Things-Need for a new legal environment", J. Computer Law \& Security Review, vol. 1, (2009), pp. 522-527.

[9] T. Wu, "The Security Problem of the Internet of Things is Analyzed", J. Network Security Technology \& Application, vol. 1, (2010), pp. 7-8.

[10] A. Perrig, "SPINES, security protocols for sensor networks", J. Wireless Networks, vol. 8, (2002), pp. 521-554.

[11] W. Zhenqiang, Z. Yanwei and M. Jianfeng, "A Security Transmission Model for Internet of Things", J. Chinese Journal of Computers, vol. 34, (2011), pp. 1351-1364.

[12] Y. Geng, X. Jian, C. Wei, Q. Zhenghua and W. Haiyong, "IOT security features and key technology", J. Journal of Naming University of Posts and Telecommunications (Natural Science edition), vol. 30, (2010), pp. 20-29.

[13] S. Jianhua, M. Chuanxiang and H. Xiaobing, "Wireless Sensor Network Routing Attack and Guard Against", J. Journal of Hubei University (Natural Science), vol. 29, (2007), pp. 355-358.

[14] L. Yanbing, H. Wenping and D. Liang, "Network information security architecture based on Internet of Things”, J. ZTE Technology Journal, vol. 17, (2011), pp. 17-20. 
[15] Y. Li, M. Jianfeng and Z. Jianming, "Trusted and anonymous authentication scheme for wireless networks", J. Journal on Communications, vol. 30, (2009), pp. 29-35.

[16] H. Fan, H. Xubin and X. Zhiyong, "Role-base access control", J. Mini-Micro syetems, vol. 2, (2000), pp. 198-200.

[17] C. Tianjie and Z. Yongping, "Role-base access control in management information system", J. Computer Applications, vol. 21, (2001), pp. 21-23.

[18] L. Yuan, Z. Wei and W. Zhixue, "Embedded Remote monitoring system based on B /S and C /S structure", J. Instrument Technique and Sensor, vol. 1, (2008), pp. 39-41.

[19] R. S. Sanhu, E. J. Coyne and H. L. L. Feinstein, "Role-base access models", J. EIII Computer, vol. 29, (1996), pp. 38-47.

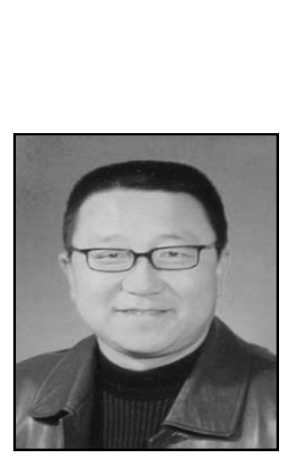

\begin{abstract}
Authors
Jia Jiang, master's tutor, is the head of Logistics Engineering Department and the director of Modern Logistics and Supply Chain Management Institute of Hebei University of Science and Technology. He has worked in the university since 1989. He is also the tobacco logistics expert of State Tobacco Monopoly Administration, the advisory expert of China International Engineering Consulting Corporation. He teaches Logistics, Supply Chain Management, Transportation and Packaging for graduate students and undergraduate students. He has finished many research programs in the national and provincial level, e.g. the National Science-supporting program. He has published many articles on International meetings and important journals as LISS、ICMLC、 Computer Science, etc.
\end{abstract}

Donghai Yang, he is a postgraduate student of Hebei University of Science and Technology. He is in the research of Logistics Service System Optimization and internet of things.

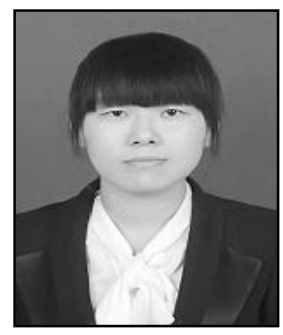

Zhe Gao, She is a postgraduate student of Hebei University of Science and Technology. She is in the research of Logistics Service System Optimization. 
International Journal of Grid Distribution Computing Vol. 8, No.4, (2015) 